\title{
Investigation on Diesel Engine Fault Diagnosis Based on Winger Spectrum Singular Value Entropy
}

\author{
Ning Sun ${ }^{1,2, * *}$, Hongmao Qin*, ${ }^{3}, *$, Siqi You ${ }^{4}$ and Ruhai Ge ${ }^{1}$ \\ ${ }^{1}$ Jiangsu University, Zhenjiang, Jiangsu, 212013, China \\ ${ }^{2}$ Society of Automotive Engineering of China, Beijing, 100055, China \\ ${ }^{3}$ State Key Laboratory of Automotive Safety and Energy, Tsinghua University, \\ 100084, China \\ ${ }^{4}$ Mechanical and Areospace Engineering, University of Missouri-Columbia, \\ MO65201, USA \\ * Corresponding Author: Hongmao Qin: qinhongmao@tsinghua.edu.cn \\ **These authors contributed equally to this work
}

\begin{abstract}
In order to quantitative description wear degree between diesel engine cylinder and piston, a fault diagnosis method based on Winger distribution combining with information entropy theory is proposed. On the basis, identification of diesel engine wear degree is taken as example. Firstly, Winger spectral is used for analysis to acquire the frequency distribution of original signal, then time-frequency entropy of Winger spectrum is established based on the information entropy theory, and characteristic parameters reflecting fault status are obtained; finally, the wear degree is judged by the size of timefrequency entropy of Winger spectrum. Experimental results show that, time-frequency entropy of Winger spectrum can identify the fault status of transmission wear degree of cylinder - piston effectively, and provide a theoretical basis for predictive diesel engine.
\end{abstract}

Keywords: fault diagnosis; Winger distribution; Singular value decomposition; information entropy

\section{Introduction}

With the progress of industrialization, machines have the tendency to become increasingly automated, electronized and even informationized. Thus, the technologies of machine monitor and fault diagnosis are highly valued in today's industry. Diesel engine as one of the classic power machines has the ability to perform reciprocating and rotary motions Due to the complication of its internal structure, multiple vibration excitation sources and interference made the vibration signal to have a non-stationary and non-linear time-varying characteristic, which cannot be used to analyze the condition of the diesel engine. Therefore, the key issue of nondestructive inspection of the diesel engine is how to extract the characteristic surface vibration signal from the pool of surface signals.

Typical nondestructive inspection of diesel engine nowadays starts with a research on the character extraction and pattern recognition theories of non-stationary and non-linear vibration signals. The process works by reprocessing the analysis on diesel engine vibration signals, extracting surface features that can represent the working conditions of the diesel engine, and comparing and categorizing these features using pattern recognition processors in order to determine the working condition and fault type of the diesel engine.

When a diesel engine is faulty, energy of vibration signal changes with the degree of fault; using the vibration signal is one of the main strategies to check for fault. There are two types of conventional signal processing: time-domain analysis and frequency domain analysis. Time domain analysis is the simpler and more efficient type. It uses the time- 
domain vibration signal for analysis. The time-domain analysis mainly includes probability analysis, time-domain synchronous averaging method, correlation function diagnoses, etc. The main focuses of time-domain analysis are vibration amplitude, vibration period and frequency, phase, skewness and kurtosis, etc. As for frequencydomain analysis, it is mainly based on Fourier transform, including spectral analysis, cepstrum analysis, envelope analysis, etc.

Traditional signal processing technologies are highly developed in theories and applications; however, it can only be used in smooth and steady signal processing. For non-smooth and non-steady signals, a more comprehensive strategy is needed for the purpose of signal analysis. Current research is focused on an analytical strategy that combines time-domain and frequency-domain analyses together (time-frequency analysis), which means to extract and analyze vibration time and frequency data simultaneously. This kind of analysis has two categories: linear and non-linear. Linear time-frequency analysis includes wavelet analysis, the short-time Fourier transformation (STFT) and Gabor expansion. As for non-linear time-frequency analysis, the quadratic linear time-frequency distribution is the most widely used type.

Wavelet analysis [1-2] is a time-varying signal processing strategy. It can categorize the target signals into different scales of wavelet transformation coefficients in order to analyze and reconstitute the signals. This way, signals can be compressed, be tested for singularity and the noises can be eliminated. Wavelet analysis has a strong advantage in dealing with non-smooth, non-linear signals; therefore, it is widely used in machine fault diagnosis.

Donoho [3] proposed a threshold noise reduction strategy in his research. Threshold can be divided into hard threshold and soft threshold. By quantizing threshold to smoothing the noise signal, the goal of noise minimization can be achieved. Lin et al. [4] came up with a continuous wavelet transform noise deduction method based on Morlet wavelet basis. It can extract periodical impulse signal completely from a gear case vibration signals that have a lot noise. Chen et al. [5] proposed block threshold signal noise minimization strategy and successfully applied it in machine error diagnosis. Kankar et al. [6] used minimum Shannon entropy criterion to extract statistical characteristics of bearing fault from discomposed wavelet coefficient, and used the characteristics as the input vector of the classification recognizer to investigate faults. In 1992, Mallat noticed the differences in propagation characters between the modulus maxima of noise elements and signal elements and proposed a wavelet de-noising strategy based on the modulus maxima [7]. By removing the non-transmissible maximum value and use alternating projection method to reconstitute the remaining modulus maxima, it is possible to reduce noise signals. Based on Mallat's research, Zhang et al. [8] provided a noise reduction algorithm using the differences in propagation characters of modulus maxima of the wavelet transform of noise and signals on different scales. This algorithm has proven to be effective and feasible. In 2002, Zheng [9] in his doctoral dissertation proposed an automatic noise reduction algorithm that focused on energy based on wavelet packet analysis. He also proposed a gear fault signal extraction strategy based on wavelet packet energy distribution. By denoising the gear fault signals, the effectiveness of this method has been proved. However, it also shows the limitation of this method. In 2010, Fan [10] used wavelet packet soft threshold denoising method in the noise reduction of non-linear electrocardiosignal and he was able to obtain a good denoising effect. In 2011, Tian et al. [11] used self-adapted wavelet packet threshold analysis in voice noise reduction. Compared to conventional algorithms used in low signal to noise ratio (SNS), their method is more effective in reducing reconstitution of signal envelope distortion and this method also enhanced the output SNS. In 2012, Chen et al. [12] used wavelet packet combined with support vector data to denoise bearing vibration signal of sorting machine, they sorted out characters like the failure frequency and wavelet packet energy and input them into a recognizer. The failure recognition rate of their method is above $98 \%$. Based 
on Donoho's theory, Yu et al. [13] proposed an advancement on the wavelet packet analysis. This advancement overcame the limitations of soft and hard threshold and has a better noise reduction ability than wavelet packet analysis.

Wavelet noise reduction method has proven to be the best in separation of complex signal and noise in multiple researches [14-23]. It has been widely used in fault diagnosis of diesel engine. [14-23]. However, classical wavelet noise reduction has its limitations [24-25]. Diesel engine has a wide vibration signal band, but wavelet transform cannot redisintegrate the details of signals. Moreover, the choices of suitable wavelet basis and correct quantization threshold value, especially for weak jump signal in strong noise, are all obstacles in noise reduction.

Winger distribution as one of the time-frequency analysis method is widely used in the analysis of non-smooth and non-steady signals because of its simple form and explicit physical significance. Therefore, a vibration signal fault recognition method based on singular value entropy of Winger spectrum is proposed in this study. This method works by obtained the Winger time-frequency spectrum of the complete time frequency surface signal distribution, and then compute the singular value entropy of Winger spectrum to diagnose the working condition and degree of fault of the diesel engine.

This paper is organized as follow: It starts by introducing some basic theories of timefrequency analysis that are going to be mentioned in the main body of this paper, including Winger distribution, singular value decomposition and information entropy. Afterwards, this a basic process of diesel engine fault diagnosis based on Winger spectrum singular value entropy is going to be established. Using the method proposed, fault diagnosis was performed on 6BB1 diesel engine for the classical piston-cylinder wear. A conclusion is provided at the end of the paper.

\section{Basic Theory}

\subsection{Winger Distribution}

Due to the fluctuation of vibration signal, the joint distribution of the time and frequency of the signal which is called the time frequency representation of the signal is needed .As a tool for time-frequency analysis, Winger distribution is widely used. For time domain signals $f(t)$, Fourier transforming to $F(\omega)$, the definition of the Winger distribution [25] is

$$
W(t, \omega)=\int_{-\infty}^{+\infty} f^{*}\left(t-\frac{\tau}{2}\right) \cdot f\left(t+\frac{\tau}{2}\right) e^{-j \omega \tau} d \tau
$$

Or

$$
W(t, \omega)=\frac{1}{2 \pi} \int_{-\infty}^{+\infty} F^{*}\left(\omega+\frac{\theta}{2}\right) \bullet F\left(\omega-\frac{\theta}{2}\right) e^{j t \theta} d \theta
$$

Where ${ }^{t, \tau}$ is time ; $\omega$ is angular frequency, $f(t)$ represents time-domain signal ; $f^{*}(t)$ is the conjugate complex numbers of $f(t) ; F(\omega)$ is the Fourier expression of signal $f(t) ; F^{*}(\omega)$ represents conjugate complex numbers of $F(\omega) ; \theta$ is phase position. Winger spectrum meet the energy integration.

$$
\begin{aligned}
& \frac{1}{2 \pi} \int_{-\infty}^{+\infty} \omega(t, \omega) d \omega=|f(t)|^{2} \\
& \int_{-\infty}^{+\infty} \omega(t, \omega) d t=|F(\omega)|^{2}
\end{aligned}
$$


Where $\omega(t, \omega)$ represents energy distribution density of time $t$; frequency $\omega$.

Therefore, Winger spectrum is the energy distribution in time and frequency plane of time-domain signal, and the Winger distribution analysis has explicit physical meaning.

\subsection{Singular Value Decomposition}

Singular value decomposition is an important matrix decomposition, the stability of singular value after decomposition is main advantage, that is, the change of singular value is not obvious when some of elements in matrix change. Besides, the characteristics of the singular value decomposition are the rotation non-deformation and proportion nondeformation.

$$
\begin{gathered}
\text { Matrix } A \in R^{m \times n} \text {, then there is an orthogonal matrix } \\
\qquad \begin{array}{r}
U=\left[u_{1}, u_{2}, \ldots, u_{m}\right] \in R^{m \times m} \\
V=\left[v_{1}, v_{2}, \ldots, v_{n}\right] \in R^{n \times n}
\end{array} \\
\text { Make } U^{T} A V=\operatorname{diag}\left[\sigma_{1}, \sigma_{2}, \ldots, \sigma_{p}\right]=S \text {,that is } \\
A=U S V^{T}
\end{gathered}
$$

Then Eq. 7 is singular value decomposition of matrix A.

$\sigma_{1} \geq \sigma_{2} \geq \ldots \geq \sigma_{p} \geq 0, \sigma_{i}(i=1,2, \ldots, p)$ where $U$ and $V$ are singular vectors of A.

Singular value decomposition ${ }^{[27]}$ is a kind of important matrix decomposition in the matrix theory which has good stability, that is to say, the change of singular value is not obvious when some of elements in matrix change.

\subsection{Information Entropy}

Information entropy can be used to reflect the uniformity of probability distribution ${ }^{[26]}$, and its mathematical expression is:

$$
s(E)=-k \sum_{i=1}^{N} E_{i} \ln E_{i}
$$

$E\left(E_{1}, E_{2}, \ldots, E_{n}\right)$ is an uncertain probability distribution, $k$ represents arbitrary constant. Information entropy has the properties of continuous non negative, extended, additive and so on. Maximum entropy is obtained when $E_{1}=E_{2}=\ldots=E_{n}$.

The value of information entropy can be used to describe the average degree of uncertainty of system. The greater the uncertainty of the system variables are, the greater the entropy is, on the contrary, The more ordered a system is, the lower the information entropy is. The above analysis showed that if the probability distribution of the system is uncertain, the entropy will be max.

\section{Diesel Engine Fault Diagnosis Based on Winger Spectrum Singular Value Entropy}

The flow chart of diesel engine fault diagnosis is shown in Figure 1. 


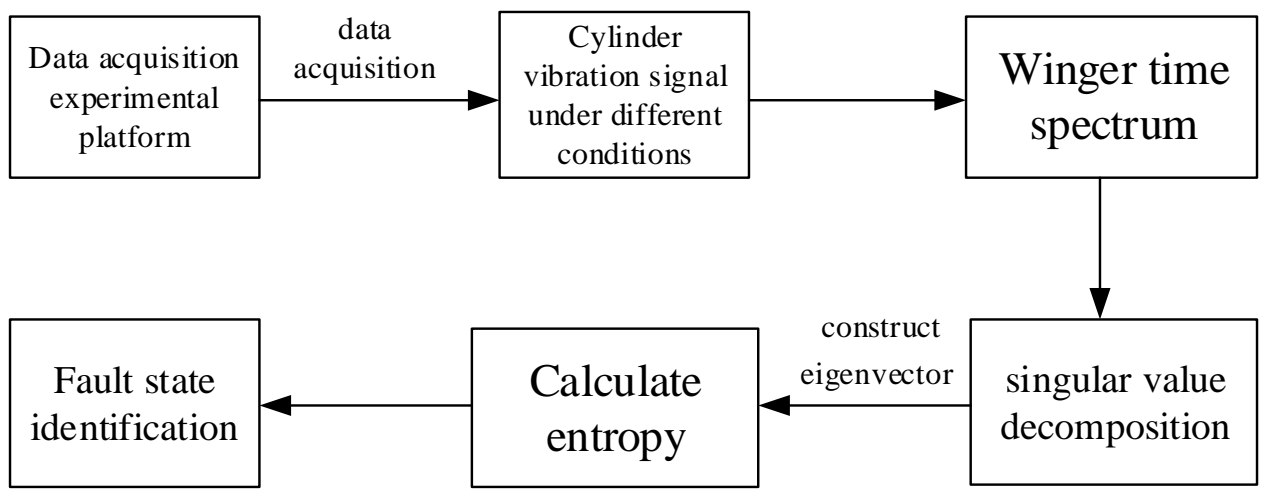

Figure 1. Diesel Engine Fault Diagnosis

The specific steps are as follows:

Different degrees of fault of diesel engine vibration signals are collected and then Wigner distribution analysis are conducted. Winger time-frequency spectrum contains a wealth of time and frequency information, the difference between time the frequency spectrum reflect the energy change in corresponding frequency, assuming Winger timefrequency matrix as A.

In this paper, the singular value decomposition of Winger time-frequency matrix $\mathrm{A}$ is carried out, and the characteristic value sequence of the mechanical state is obtained. The singular value in all frequency domain reflect the difference of different fault condition, in order to quantitatively describe the degree of this change, we will introduce the theory of information entropy.

Singular value $R_{1}^{2}, R_{2}^{2}, \ldots, R_{n}^{2}$ can be obtained by singular value decomposition on timefrequency matrix A. Each component is normalized to obtain $E_{i}=\frac{R_{i}^{2}}{E}$, where $E=R_{1}^{2}+R_{2}^{2}+\ldots+R_{n}^{2}$, then there is $\sum_{i=1}^{n} E_{i}=1$,This confirms with Initialization conditions for the calculation of information entropy.

the singular value entropy is constructed according to the definition of information entropy, the formula for calculating the time-frequency entropy of Winger spectrum is [28-29]:

$$
S(E)=-\sum_{i=1}^{N} E_{i} \ln E_{i}
$$

According to the properties of entropy, the uncertainty of the system is large that the value of $S(E)$ is large when the distribution of $E_{i}$ is uniform, otherwise, value of $S(E)$ is small. Therefore, the Winger spectrum singular value entropy can be used to identify the fault degree of the diesel engine under different working conditions. Experimental analysis will be conducted based on Winger spectrum singular value entropy theory.

\section{Application}

Piston - cylinder liner wear is one of the common faults of diesel engine. The cause is that the larger piston side thrust produced great impact force, impact which take place in the main thrust face happens from one side of the cylinder to the other side, impact on the cylinder liner transfers to the cylinder surface in the form of vibration signal. Along with the increasing of the piston cylinder liner wear, the energy of the vibration signal which is 
reflected to the surface of the cylinder is larger, and the signal noise ratio is improved by means of reasonable noise reduction, which is convenient for signal analysis and fault diagnosis. [30-32]

Figure 3 is the time domain wave of vibration signal after noise reduction which is extracted from 6BB1 diesel engine under different wear conditions, where sampling frequency is $25.6 \mathrm{kHz}$ and the number of sampling points are 1024.Table 1 shows original parameters of the cylinder and the actual test data under four kinds of States.

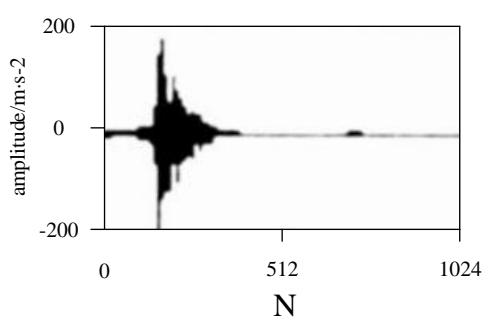

(a)normal condition ( $\mathrm{NC}$ )

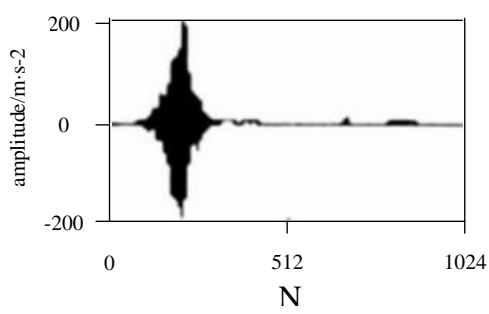

(c)moderate wear (MW)

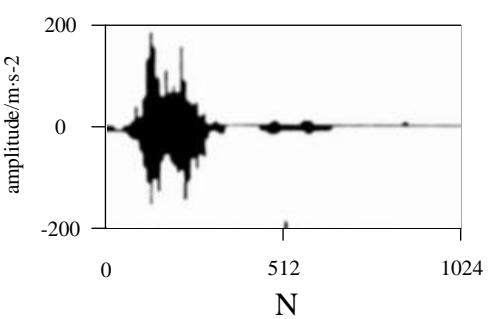

(b)slight wear ( SW )

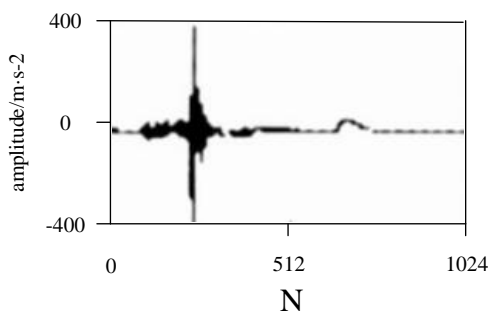

(d)heavy wear (HW)

Figure 2. Time Domain Waveform of Surface Vibration Signal in Different States

Table 1. Original Parameters and Measured Data of Piston - Cylinder Liner (Mm)

\begin{tabular}{cccc}
\hline state & $\begin{array}{c}\text { tolerance clearance } \\
\text { between piston and } \\
\text { cylinder. }\end{array}$ & $\begin{array}{c}\text { diameter of } \\
\text { cylinder liner } \\
\text { variation }\end{array}$ & $\begin{array}{c}\text { Piston } \\
\text { diameter } \\
\text { variation }\end{array}$ \\
\hline $\begin{array}{c}\text { factory standard } \\
\text { utilized limit } \\
\text { normal }\end{array}$ & $0.182 \sim 0.20$ & $0 \sim 0.02$ & $-0.025^{\sim} 0$ \\
condition & 0.580 & 0.200 & -0.250 \\
slight wear & 0.205 & 0.024 & -0.028 \\
moderate wear & 0.325 & 0.095 & -0.077 \\
heavy wear & 0.485 & 0.174 & -0.158 \\
\hline
\end{tabular}

It can be seen from Table 1 that the piston cylinder liner tolerance clearance increases when piston cylinder liner wear become severe at the same time, which leads to the obvious decrease of air tightness and explosion pressure, and the collision between piston and cylinder liner increase.

Figure 3 shows Winger time spectrum of vibration signal in different states. As is demonstrated in the figure the vibration signal energy is relatively concentrated under normal conditions, But the signal energy diffuse to broadband range gradually with 
increase of the degree of fault, and duration is reducing. The change trend of energy can be obtained by Winger time frequency spectrum. In order to quantitatively describe vibration signal Winger time-frequency feature, the calculation of Winger spectrum singular value entropy is conducted by the method proposed in this paper, and comparison of time and frequency domain information entropy of combustion stage is carried out. The calculation results are shown in Figure 4.
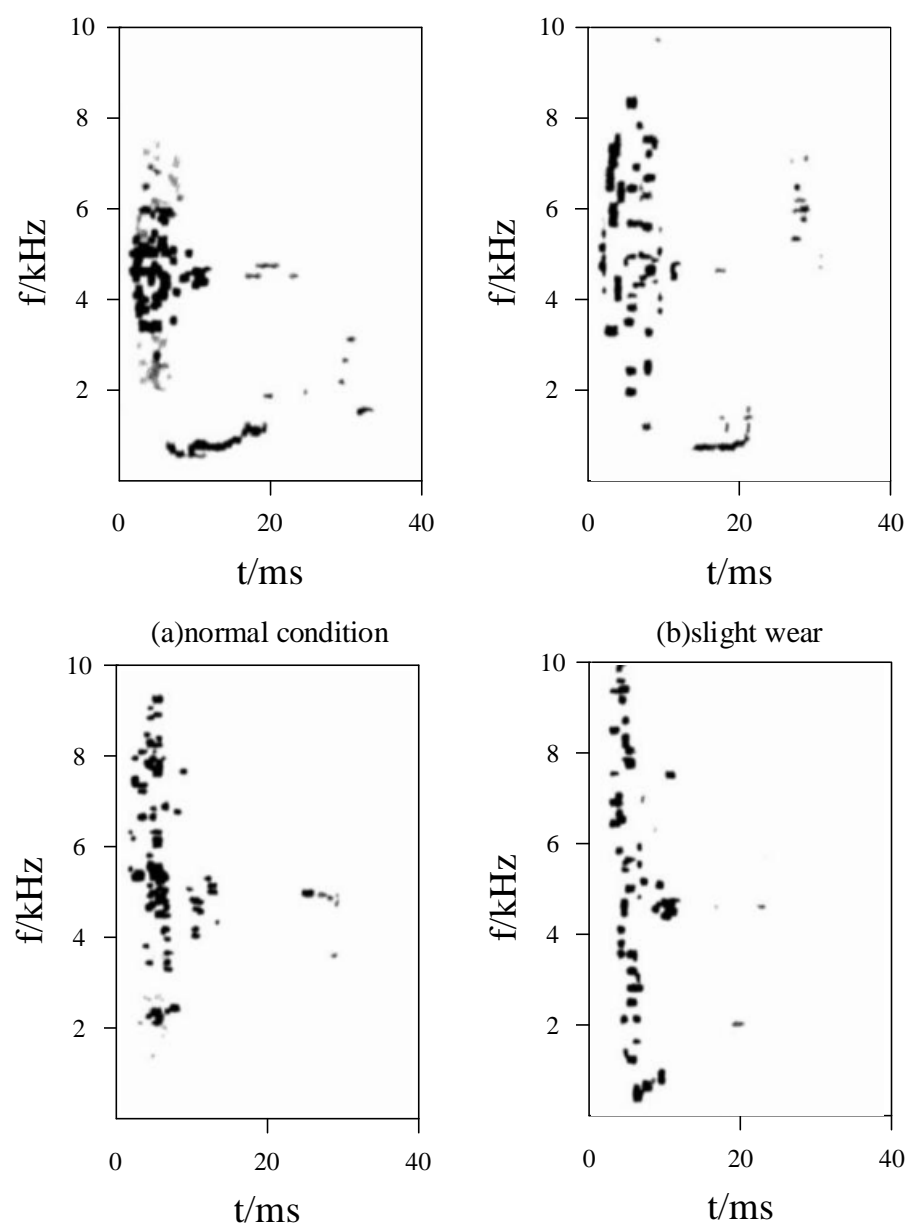

(c)moderate wear

(d)heavy wear

\section{Figure 3. Winger Time Spectrum of Vibration Signal in Different States}

As shown in Figure5, time and frequency domain information entropy can't clearly describe the degree of wear between the piston and cylinder liner. The information entropy under moderate wear is larger than it under heavy wear, which is mainly due to diesel engine vibration signal is a complex non-stationary signal composed of many exciting source. The time or frequency domain information under the single state is not able to completely describe the vibration signal. Entropy $\mathrm{S}$ is determined by Wigner spectrum singular value entropy theory. The result shows that information entropy increase with rising of degree of fault, corresponding to the energy distribution in timefrequency analysis which is consistent with the maximum entropy principle. It means Winger spectrum singular value entropy can be used to quantitatively describe the degree of wear between piston and cylinder liner and identify the fault state of diesel engine. 


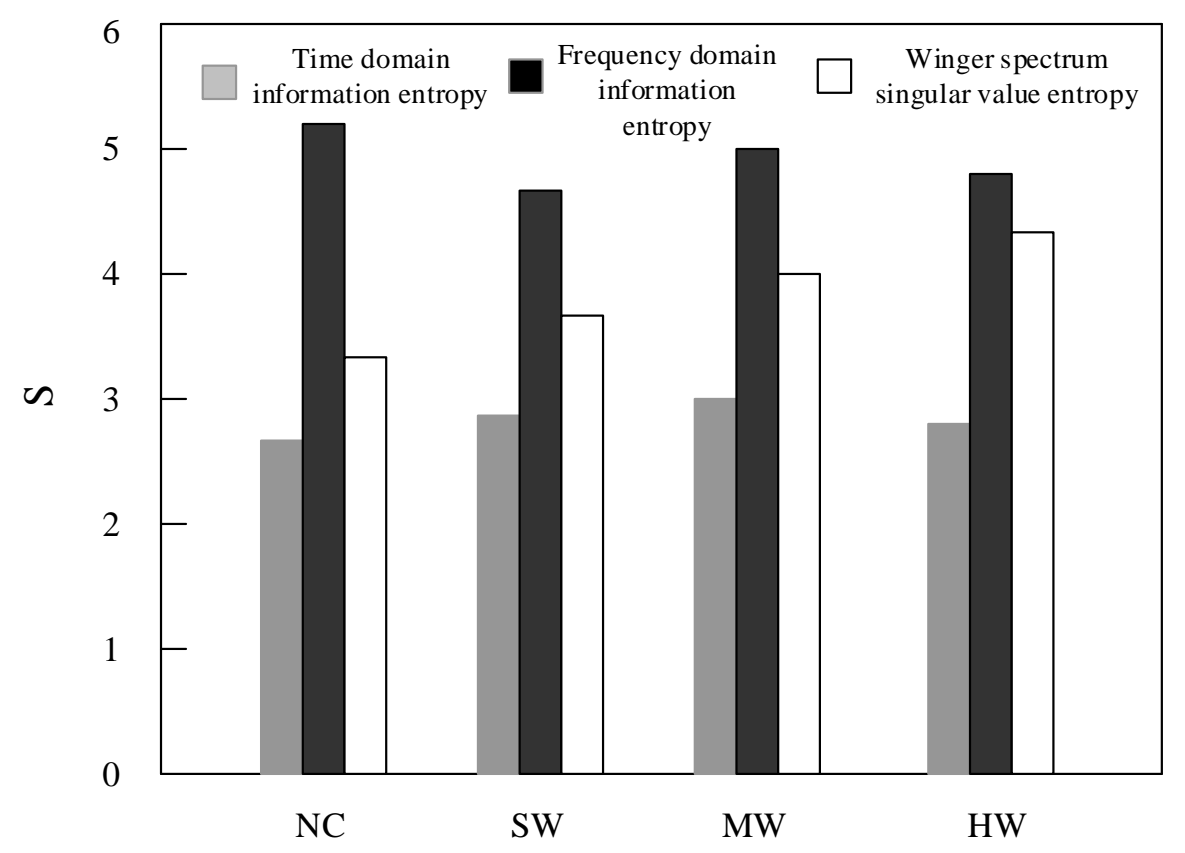

Figure 4. Comparison between the Calculated Information Entropy

Based on the size of gap and value of corresponding entropy, fitted curve is obtained in the help of least square method as shown in Figure5 where abscissa is piston - cylinder liner clearance and value of ordinate is entropy. The relational expression is:

$$
y=0.3268 S-0.9395
$$

Where $S_{\text {represents entropy, }}{ }^{y}$ is piston - cylinder liner clearance.

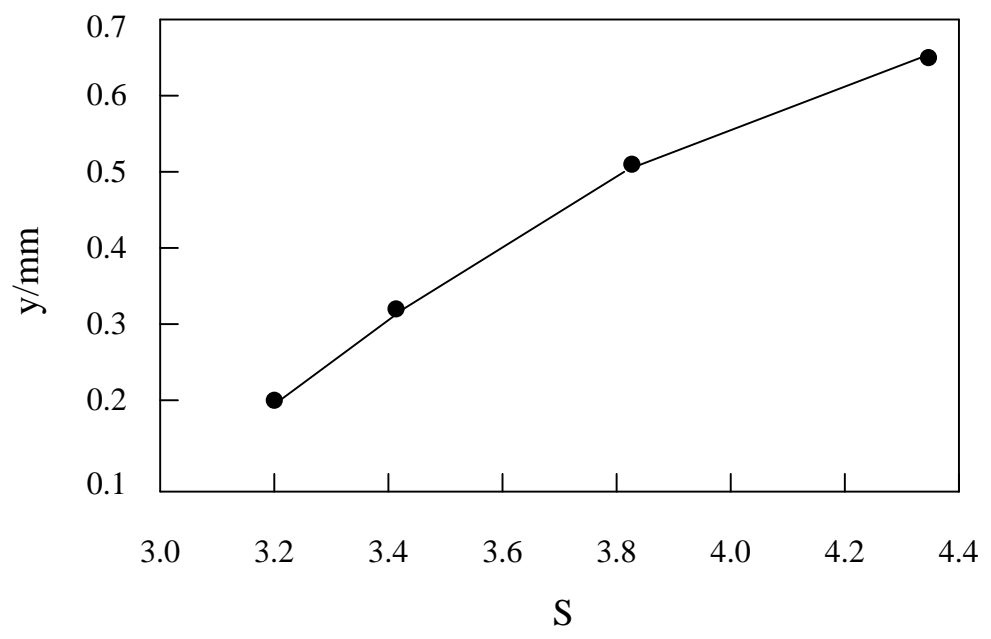

Figure 5. The Relationship Between Winger Spectrum Singular Value Entropy and Clearance

Piston - cylinder liner clearance can be derived by entropy so as to determine the degree of fault based on the corresponding relationship between entropy and clearance established by least square method. Winger singular spectrum entropy value can be used to primarily assess the state of diesel engine which not only is a good foundation of diesel 
engine intelligent fault diagnosis, but also prove the feasibility of diesel engine fault diagnosis based on Wigner spectrum singular value entropy.

\section{Conclusion}

A fault feature extraction method based on Winger spectrum singular value entropy is proposed in this paper. The Winger spectrum singular value entropy can effectively reflect the clearance between piston and cylinder liner by analyzing the vibration signal of diesel engine, so as to make a preliminary diagnosis of the fault degree. The quantitative description of energy distribution and state evaluation are given by fitted curve in the help of least square method. To realize the fault identification of diesel engine of different degree has reference value for other complex mechanical equipment fault diagnosis.

\section{Acknowledgments}

The research was supported by the Jiangsu provincial key laboratory of new technology application on road delivery vehicle (NO: BM20082061501), and the National Natural Science Foundation of China under Grant 51505247. And the Corresponding Author is Hongmao Qin (Email: qinhongmao@tsinghua.edu.cn).

\section{References}

[1] Chen F ,Tang B ,Zhao L.A filtering method based on optimal morlet wavelet and its application in machine fault feature analysis[J].China Mechanical Engineering, (2008),19(12):1437-1441.

[2] CuiL,GaoL,YinH,etal.Research on Composite Fault Diagnosis Method Based on the Second Generation Wavelet[J].China Mechanical Engineering, (2008), 20(4): 442-446

[3] D.L.Donoho. De-noising by soft-thresholding. IEEE Transactions on Information Theory, (1995), 41(3):613 627.

[4] Lin Jing, Q.L. Feature extraction based on Morlet wavelet and its application for mechanical fault diagnosis. Journal of Sound and Vibration, (2000),234(1):135 148

[5] Chen Z, Xu J, Yang D. Noise reduction method based on repeated wavelet packet threshold value and its application in machine fault diagnosis, Journal of Mechanical Engineering, 2007(06):200 204

[6] P.K.Kankar, Rolling element bearing fault diagnosis using wavelet transform. Neurocomputing. 2011(74):1638 1645

[7] MallatS,Hwang W L. Singularity Detection and Processing With Wavelets. IEEE Trans Information Theory, 1992,38(2):617 643

[8] Zhang Y, Research on Signal Noise Reduction Based on Wavelet Transform modulus maxima, Journal of Agricultural University of Hebei, 2009,32(1):114 116

[9] Zheng H,Study on Characteristic Signal Extraction of Non-smooth Non-steady Non-linear NonGaussian Signals and Fault Diagnosis Method: [Doctoral Thesis Academic Dissertation], HeFei University of Technology,2002.6

[10] Fan Y, Application of Wavelet and Wavelet Packet in the Noise Reduction of Electrocardiosigna, Journal of Chongqing University of Science and Technology (Natural Science Edition), 2010,2,12(1): $128 \sim 130$

[11] Tian Y, New Voice Noise Reduction Algorithm on Wavelet Packet Automatic Threshold Value, Applied Acoustics, 2011,1,30(1):72 79

[12] Chen W, Jia Q, Sun H, Sorting Machine Bearing Fault Diagnosis Based on Wavelet Packet and SVDD, Journal of Vibration, Measurement \& Diagnosis,2012,10,32(5):762 766

[13] Yu W, Zhang Q, Wavelet Packet Noise Reduction Based on Advanced Threshold Noise Reduction Algorithms. Communications Technology, 2010,0643(222):7 9

[14] MaXiaoyan, Signal Reconstruction Based on Mean Threshold Wavelet Packet De-noising. Proceeding of ICSP 2000,387 390

[15] Quan Pan, Two denoising method by wavelet transform. IEEE Transaction on Signal Proceccing, 1999,47(12):567 589

[16] Sumit K., Nath, Wavelet based compression and de-noising of optical tomography data. Optics Communications, 1999,167:37 46

[17] Wang G, Application of Time-Frequency Analysis in Machine Fault Diagnosis. Journal of Dalian University of Technology, 1996,36(3):301 305

[18] Shi D, Qu L, Application of Self-adapted Time-Frequency Analysis in Rotary Machine Fault Diagnosis. Journal of Vibration Engineering,2000,13(2):271 276 
[19] Zhang J, Zhang Y, Xie Y, Research on Application of Time-Frequency Analysis in Early Diagnosis of Impact Fault, Journal of Vibration Engineering,2000,13(2):222 228

[20] Liu S, Application of Advanced Wavelet Packet Algorithms in Vibration Diagnosis, Transactions of CSICE, 2000,18(1):11 16

[21] Peng Z, He Y, Zhu F, Research on the Application of Wavelet Scale Spectrum in Vibration Signal Analysis, Journal of Mechanical Engineering,2002,38(3):122 126

[22] Gary G. Yen, Kuo-Chung Lin. Conditional health monitoring using vibration signatures. Proceedings of the 38th Coference on Decision \&Control ,1999:4493 4498

[23] Hao Ye, Ping Zhang, A time-frequency domain fault detection approach based on parity relation and wavelet transform. Proceedings of the 39th IEEE Conference on Decision and Control,2000:4156 4161

[24] Chen D, Cao C, Peng W, Wavelet Time-Frequency Analysis in Engineering Signal, Journal of Chongqing University of Science and Technology (Natural Science Edition), 1999,22(5):27 31

[25] Chen X, Chen J, Presseseccentric Rotating Stall Portent Time-Frequency Analysis Based on Wavelet Transform, Journal of Engineering Thermophysics, 2000,21(3):289 293

[26] CaiY,LiA,ShiL, Ic engine diagnosis method based on emd-wvd vibration spectrum time-frequency image recognition by SVM[J].Chinese Internal Combustion Engine Engineering, (2012),33(2):72-78.

[27] ChenX,YuD,LuoJ.Gear tooth fault diagnosis by using order cyclostationary demodulating approach Based on chirplet Path Pursuit[J].Journal of Mechanical Engineering, (2012),48(3):126-130.

[28] DingK,LiW,Zhu X. Gear and gear box fault diagnosis operative technology[M].Bei Jing: China Machine Press, 2005.

[29] [CANDES E J,CHARLTON P R,HELGASON H, Detecting highly singnals by chirplet path pursuit[J].Applied and Computational Harmonic Analysis, (2008),24(1):14-40.

[30] SunY,LiuB,CuiT, Model-based fault diagnosis method of diesel engine intake system[J]. Vehicle Engine, (2013), 18(3):84-87.

[31] ZhaoZ.Research on vibration signal based machinery fault feature extraction and diagnosis [D].BeiJing:BeijingJiaotong University, (2013). 